\title{
Numerical Simulation of Sintering of Non-equal Metal Powders by Surface Diffusion
}

\author{
Song Min', Zheng Zhoushun', Chen Dongdong ${ }^{1}, \quad$ Tang Huiping $^{2}, \quad$ Wang Jianzhong ${ }^{2}$ \\ ${ }^{1}$ Central South University, Changsha 410083, China; ${ }^{2}$ State Key Laboratory of Porous Metal Materials, Northwest Institute for Nonferrous \\ Metal Research, Xi'an 710016, China
}

\begin{abstract}
This paper focuses on the numerical simulation of sintering of non-equal metal powders by surface diffusion. In order to simulate the sintering process of non-equal metal powders clearly, the surface diffusion model was modified by establishing more suitable initial boundary conditions. And this model was numerically solved by the finite difference method. The 2D and 3D numerical simulations of two metal powders with radii ratios of $1: 1,1: 1.5,1: 2$ were implemented by this model. Through comparison, the simulation results are consistent with the experimental ones. Finally, the effect of metal powders' radii ratios on the growth rate of sintering neck was investigated. The results show that the sintering neck radius increases with the decreasing of radii ratios of metal powders.
\end{abstract}

Key words: sintering; metal powders; surface diffusion; finite difference; radii ratio

Mass transport occurs at high temperature with a specific atmosphere. Surface diffusion, as a mass transport mechanism, plays an important role in sintering process of metal powders, metal fibers and grain-boundary grooves ${ }^{[1-5]}$.

Sintering process have been extensively studied during the last decades ${ }^{[6-9]}$, and many numerical methods have been used to simulate it, such as the MOL method ${ }^{[10]}$, the finite element $\operatorname{method}^{[11]}$, and the level set method ${ }^{[12]}$. The surface diffusion model, established by a nonlinear partial differential equation, was firstly proposed by Mullins ${ }^{[13]}$. For obtaining nearly flat surfaces, the small-slope limit was introduced to simplify the model. Subsequently, based on the seminal work of Mullins, many researchers concentrated on the symmetric model to numerically simulate the sintering process of grain-boundary grooves and metal powders ${ }^{[14-16]}$, which means the investigation on the sintering process of symmetric system have been very mature. However, in the actual sintering process, the billet is usually composed of the powders with different dimensions. For solving this problem, some researches on the sin- tering process of non-symmetric model were proposed. Pan and Cocks ${ }^{[17]}$ analyzed the coupled surface and grain-boundary diffusion for the non-isometric model. Zhang and Schneibel ${ }^{[18]}$ investigated the sintering process of two equal and non-equal circular particles by surface and grain-boundary diffusion. Both in their research, the grain-boundary was assumed to be straight; however, the unbalanced total force in the perpendicular direction of the grain-boundary makes it impossible. Then, Du and Liang ${ }^{[19]}$ adopted a non-isometric sintering model to deal with the initial sintering process and some fixed geometrical parameters were used in their research. Compared with the actual sintering process, the sintering junction and its neighboring points are constantly changing, which means that some geometrical parameters can not be fixed. In the previous numerical simulation, a fixed angle or some fixed geometrical parameters were used to deal with the boundary, which can not describe the sintering process of non-equal metal powders exactly. To date there is no perfect numerical simulation to solve the problem. Therefore, it

Received date: October 28, 2016

Foundation item: National Natural Science Foundation of China (51174236, 51134003); The National Basic Research Development Program of China (2011CB606306); The Opening Project of State Key Laboratory of Porous Metal Materials (PMM-SKL-4-2012); Innovation Project of Central South University (2016zzts220))

Corresponding author: Zheng Zhoushun, Ph. D. Professor, School of Mathematics and Statistics, Central South University, Changsha 410083, P. R. China, Tel: 0086-731-88660152, E-mail: 2009zhengzhoushun@163.com 
is necessary to adopt a new boundary condition to describe the sintering process of non-equal metal powders clearly.

In this paper, combined the geometric structure of non-equal metal powders with the surface diffusion model, a new treatment of boundary condition was proposed. And the three-dimensional model of metal powders was developed to simulate the sintering process by surface diffusion. The $2 \mathrm{D}$ and $3 \mathrm{D}$ results of metal powders with different radii ratios were described. In addition, the effect of the metal powders' radii ratios on the growth of sintering neck was also investigated.

\section{Mathematical Model}

\subsection{Geometric model}

As shown in Fig.1, two metal powders have different radii $R_{1}$ and $R_{2}, O_{1}$ is the section of metal powder on the left, and $O_{2}$ represents the section of metal powder on the right. The two metal powders, with their neck radius $Y$, are inseparable throughout the sintering process due to mutual attraction.

\subsection{Surface diffusion model}

Surface diffusion results in mass flow along the materials surface and hence changes surface morphology ${ }^{[20]}$. According to the model derived by Mullins ${ }^{[13]}$, mass transport by surface diffusion is driven by the gradient of chemical potential which is proportional to the surface flux. And the chemical potential on the surface is proportional to the curvature, so the surface flux is proportional to the gradient of curvature. In order to establish a reasonable model to describe the sintering process, the metal powders are regarded as spheres, and the surface diffusion is assumed to be the only mechanism responsible for surface movement. In this paper, only the incipient sintering process is studied, which means the radii of metal powders are nearly changeless.

In $2 \mathrm{D}$ case, the surface is represented by a curve. The mathematical model describing the surface movement introduced by Mullins ${ }^{[13]}$ can be expressed as

$$
\frac{\partial y}{\partial t}=-B\left\{\left(1+y_{x}^{2}\right)^{-1 / 2}\left[y_{x x}\left(1+y_{x}^{2}\right)^{-3 / 2}\right]_{x}\right\}_{x}
$$

where $B$ is a coefficient defined by

$$
B=\frac{\delta_{\mathrm{s}} D_{\mathrm{s}} \gamma \Omega}{k T}
$$

where $D_{\mathrm{S}}$ is the surface diffusion coefficient, $\gamma$ is the surface free energy per unit area, $\Omega$ is the atomic volume, $\delta_{\mathrm{S}}$ represents

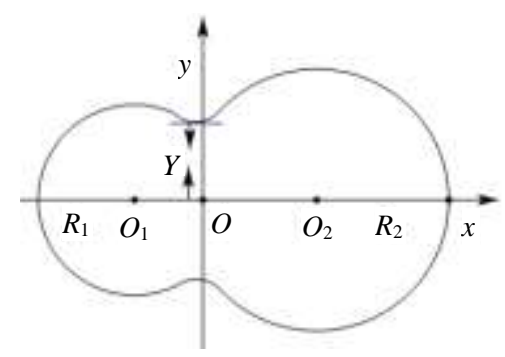

Fig.1 Geometric model of the non-equal metal powders the surface diffusive width, $k$ is the Boltzmann's constant and $T$ is the absolute temperature.

The function $y(x, t)$ is the surface profile with $t$ representing time. The curvature of surface is

$$
K=-\frac{y^{\prime \prime}}{\left(1+y^{\prime 2}\right)^{3 / 2}}
$$

which is positive for convex surfaces. The surface flux is defined as

$$
J_{s}=-\frac{\delta_{\mathrm{s}} D_{\mathrm{s}} \gamma}{k T} \frac{1}{\left(1+y^{\prime 2}\right)^{1 / 2}} \frac{\partial K}{\partial x}
$$

The characteristic length scale in the sintering process is the initial radius of metal powders $R$. A dimensionless time $t^{*}=B t / R^{4}$ and variables $K^{*}=R K, x^{*}=x / R, y^{*}=y / R$ are introduced. Substituting these dimensionless variables into Eq.(1) and Eq.(4), we have

$$
\left\{\begin{array}{c}
\frac{\partial y}{\partial t}=-\frac{\partial J_{s}}{\partial x} \\
J_{s}=-\frac{1}{\left(1+y^{\prime 2}\right)^{1 / 2}} \frac{\partial K}{\partial x} \\
K=-\frac{y^{\prime \prime}}{\left(1+y^{2}\right)^{3 / 2}}
\end{array}\right.
$$

According to the geometry of non-equal metal powders, if the boundary conditions of surface diffusion model are established, the numerical simulation on the sintering process of non-equal metal powders can be achieved.

\subsection{Boundary conditions}

The effect of surface diffusion is to make the sintering surface smooth. In the previous numerical simulation, a discontinuous point always exist at the sintering junction. For describing the sintering process more accurately, a new initial boundary condition is proposed. Let the sintering junction lie at $x=X$, using the superscripts '-' and '+' to indicate the left and right one-sided limits. In this numerical simulation, the surface at the junction is assumed to be continuous except the first time. The boundary conditions are as follows:

1) Two metal powders are inseparable throughout the sintering process due to mutual attraction. The continuity of the surface at the junction is

$$
y\left(X^{-}, t\right)=y\left(X^{+}, t\right)=Y
$$

2) In the initial state, two metal powders contact, the surface gradient is discontinuous at the junction, and the left and right one-sided limits are not equal. In this paper, at the first time, the sintering junction meets

$$
y_{x}(X, 0)=\frac{y_{x}^{-}(X, 0)+y_{x}^{+}(X, 0)}{2}
$$

Subsequently, surface diffusion will make the curve profile smooth, the surface gradient becomes continuous, and this condition is still suitable.

3) At $x=-2 R_{1}, 2 R_{2}$, according to the assumption by Mullins ${ }^{[13]}$, at the edge of the system $J_{S}=0$. In this paper, the same hypothesis is implemented. And in the initial sintering process, the condition $y\left(-2 R_{1}, t\right)=0, y\left(2 R_{2}, t\right)=0$ are assumed. 


\section{Numerical Simulation Method and Results}

\subsection{Numerical simulation method}

The finite difference method is used to solve this system numerically. In the initial stage, two metal powders contact and the surface gradient is discontinuous at the sintering junction; this moment the boundary conditions in 1.3 are used to deal with it. Since the surface diffusion is driven by the curvature of surface, the sintering process will reach to a local steady state at the second time step, that is, the sintering junction and its neighboring points have the same bending degree. This condition can be used to calculate a time step $\tau$. Subsequently, the surface gradient is continuous at the junction, and the standard finite difference can be adopted to simulate the whole process.

There are different methods in time and spatial discretization. Here a central finite difference is used to approximate the derivatives in spatial discretization, and the forward Euler difference is used to approximate the derivatives in time discretization. The details are as follows:

The first order derivative in time is:

$$
\frac{\partial y}{\partial t}=\frac{y(i, k+1)-y(i, k)}{\tau}
$$

The first order derivative in space is:

$$
\frac{\partial y}{\partial x}=\frac{y(i+1, k)-y(i-1, k)}{2 h}
$$

The second order derivative is approximated by

$$
\frac{\partial^{2} y}{\partial x^{2}}=\frac{y(i+1, k)-2 y(i, k)+y(i-1, k)}{h^{2}}
$$

where $h$ represents the spatial step, and $\tau$ is the time step.

Thus, the surface diffusion model based on the finite difference method is

$$
\begin{gathered}
y(i, k+1)=y(i, k)-\tau \frac{J(i+1, k)-J(i-1, k)}{2 h} \\
J(i, k)=-\frac{K(i+1, k)-K(i-1, k)}{2 h\left(1+\frac{(y(i+1, k)-y(i-1, k))^{2}}{4 h^{2}}\right)^{1 / 2}} \\
K(i, k)=-\frac{y(i+1, k)-2 y(i, k)+y(i-1, k)}{h^{2}\left(1+\frac{(y(i+1, k)-y(i-1, k))^{2}}{4 h^{2}}\right)^{3 / 2}}
\end{gathered}
$$

where $i=1,2 \ldots M_{1}+M_{2}+1, k=1,2 \cdots N+1, M_{1}, M_{2}$ represent the spatial steps, and $N$ represents time steps.

\subsection{Numerical simulation results}

The numerical simulation focuses a 3D study on 2D metal powders with different radii ratios $1: 1,1: 1.5$ and 1:2. Since the metal powders are regarded as spheres, the 3D simulation results are established by rotating $2 \mathrm{D}$ cross sections. Our computation is performed on Matlab, and the spatial discretization width is $h=0.02$. In this numerical simulation, the radius of the left metal powder is fixed at 1 , and the radii of the right metal powders are 1, 1.5 and 2 . The curve profile is marked at the time steps $N=1,500,50000,200000$. The 3D simulations are shown at corresponding time steps in Fig.2, Fig.4 and Fig.6, which represent different radii ratios $(1: 1,1: 1.5,1: 2)$ of two metal powders. In addition, the curves at the above-mentioned time are shown in Fig.3, Fig.5 and Fig.7.

2.2.1 2D and 3D simulation results of non-equal metal powders

As can be seen from $2 \mathrm{D}$ and $3 \mathrm{D}$ simulation results in Fig.2 Fig.7, for the non-symmetric system, the atoms of sintering metal powders make a migration to a larger curvature gradient, which leads to the irregularity of the geometric structure of sintering neck. Above phenomenon partly because of mass transport by surface diffusion is driven by the gradient of chemical potential.

In addition, two metal powders are connected to a whole one quickly in the initial state, and large numbers of atoms move to the sintering neck along the surface of metal powders, which makes the sintering neck widen quickly. Subsequently, due to the decreasing of curvature gradient, the growth rate of sintering neck goes down gradually with the holding time increasing.

Besides, for the metal powders with different radii ratios(1:1, $1: 1.5,1: 2)$, the growth trend of sintering neck are nearly identical, but their growth rates of sintering neck radii are different.
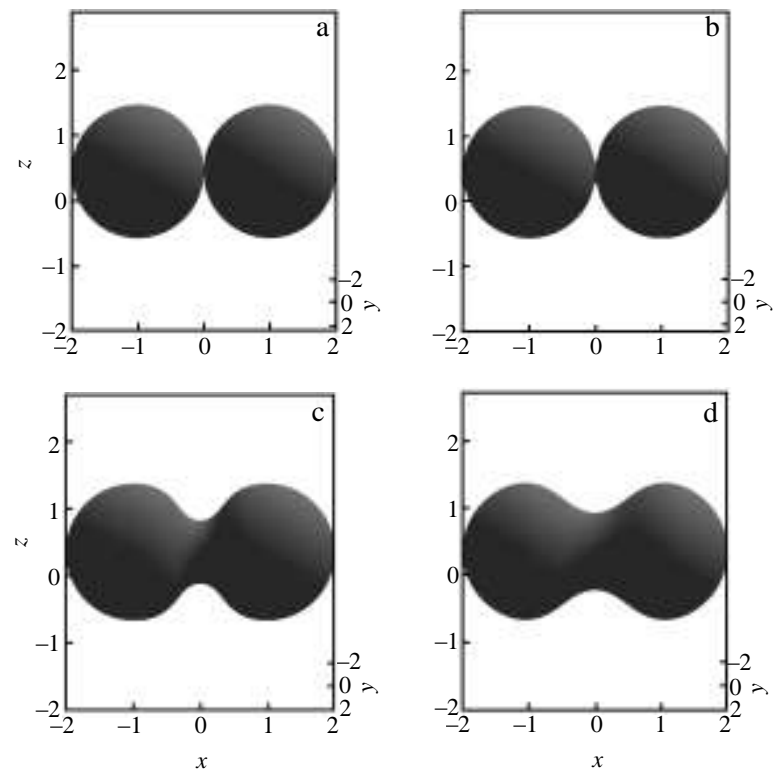

Fig.2 3D results of two metal powders with radii ratio $1: 1$, at $N=1$ (a), 500 (b), 50000 (c), and 200000 (d)

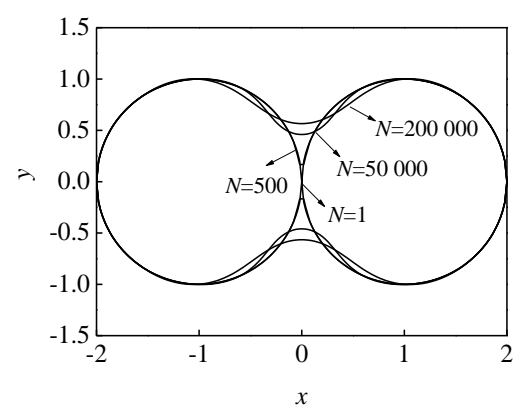

Fig.3 2D section of two metal powders with radii ratio 1:1 

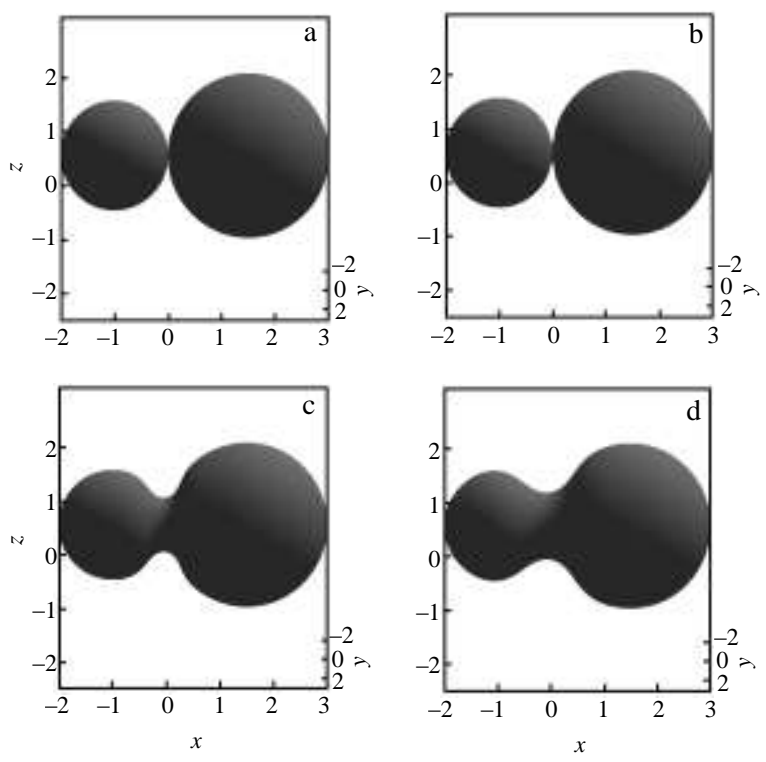

Fig.4 3D results of two metal powders with radii ratio 1:1.5, at $N=1$ (a), 500 (b), 50000 (c), and 200000 (d)

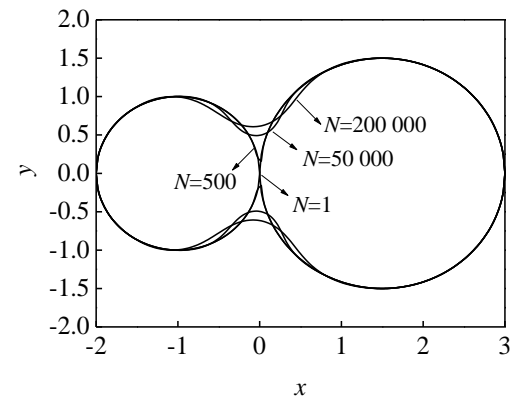

Fig.5 2D section of two metal powders with radii ratio 1:1.5
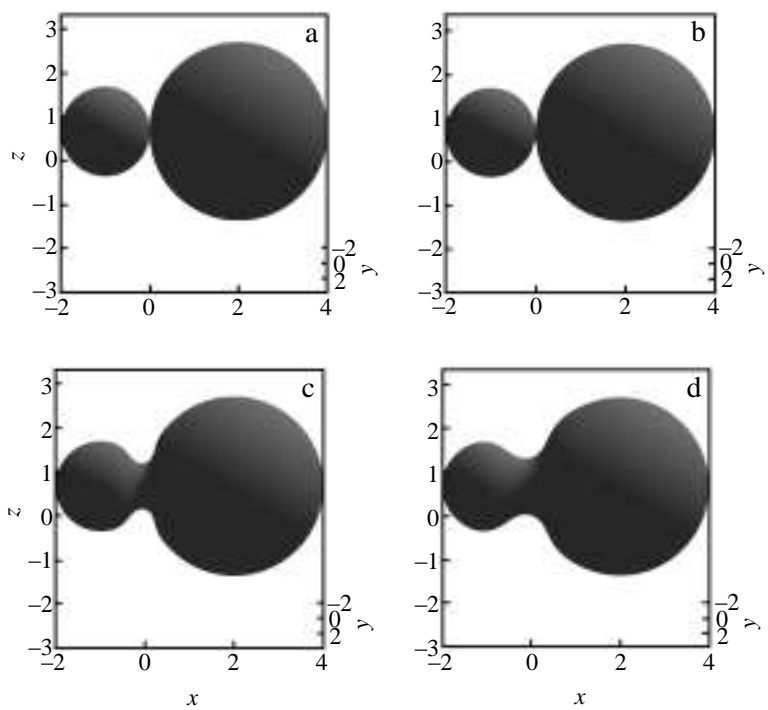

Fig.6 3D results of two metal powders with radii ratio 1:2, at $N=1$ (a), 500 (b), 50000 (c), and 200000 (d)

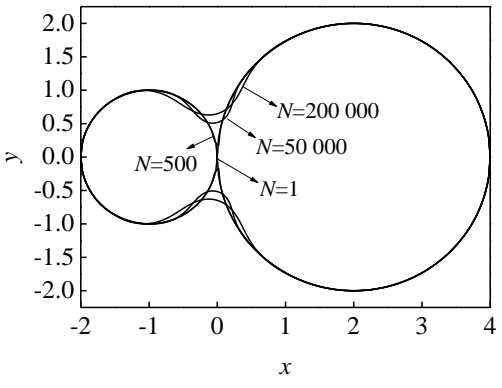

Fig.7 2D section of two metal powders with radii ratio 1:2

\subsubsection{Comparisons with experimental results}

The experimental results of sintering metal powders with different radii ratios are shown in Fig.8. Although the radii ratios are very difficult to determine exactly, comparing the numerical results (as shown in Fig.2 Fig.7) with experimental ones, it can be concluded that the numerical simulation results are well consistent with the experimental ones to some extent.

Compared with 2D and 3D simulation results shown in Fig.2 Fig.7, the crunode of metal powders changes from point contact to surface contact to form sintering neck with the holding time increasing. And for the non-symmetric system, the sintering neck is not regular. All these are consistent with the actual sintering process. Besides, in the numerical simulation, the growth rate of sintering neck is quite fast and nearly grows linearly with the time increasing in the initial stage, and then it declines gradually with the time going on. This phenomenon is very consistent with the experimental results. The reason is that, a huge concentration difference leads to the large driven force of sintering, which makes the atoms move to the sintering junction quickly and aggregate to form the sintering neck, and then the growth rate of sintering neck slows down with the decreasing of concentration difference.

Although the actual radii ratios of metal powders are very difficult to determine exactly, and the size of metal powders in the numerical simulation may be not the experimental ones, we can still draw conclusions that the numerical results are in accordance with the experimental ones and the numerical simulation reproduces the process of sintering in a certain extent.

\section{Discussion}

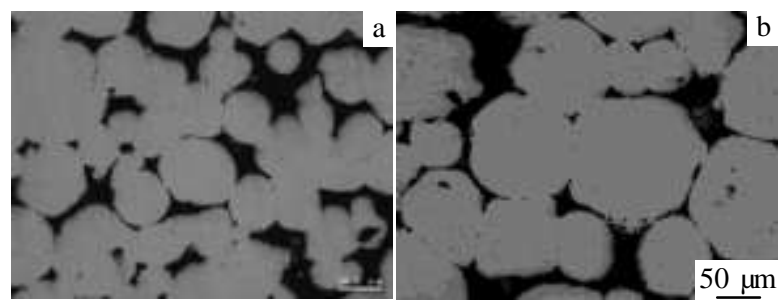

Fig.8 Experimental results of sintering metal powders 


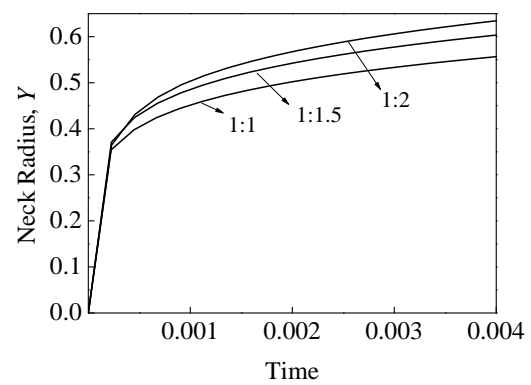

Fig.9 Growth of sintering neck radius vs time

For two non-equal metal powders with radii ratios $1: 1,1: 1.5$, $1: 2$, in order to analyze the effect of radii ratios on the growth of sintering neck exactly, the same time period is chosen. The growth of sintering neck radius is plotted as a function of time in Fig.9.

As is shown in Fig.9, at the beginning of sintering process, the effect of metal powders' radii ratios on the growth of sintering neck is not obvious. Besides, for the metal powders with different radii ratios, the growth trend of sintering neck is nearly identical. The growth rate of sintering neck is quite fast in the initial stage of sintering process, and its growth is almost linear with the time extending. Subsequently, owing to the reducing of the curvature difference and driven force, the growth rate slows down gradually with the time going on, and then the sintering process reaches to an equilibrium state. In addition, if the radius of left metal powder is fixed, with the decreasing of the metal powders' radii ratios, the growth rate of sintering neck increases.

\section{Conclusions}

1) The numerical results are in accordance with the experimental ones and the numerical simulation reproduces the sintering process in a certain extent.

2) The effect of the metal powders' radii ratios on the growth of sintering neck is analyzed. And with the decreasing of the metal powders' radii ratios, the growth rate of sintering neck increases.

\section{References}

1 Chen Dongdong, Zheng Zhoushun, Tang Huiping et al. Rare Metal Materials and Engineering[J], 2016, 45(11): 2912 (in Chinese)

2 Chen Dongdong, Zheng Zhoushun, Wang Jianzhong et al. Journal of Central South University[J], 2015, 22(7): 2446

3 Chen Dongdong, Zheng Zhoushun, Wang Jianzhong et al. Rare Metal Materials and Engineering[J], 2017, 46(6): 1474

4 Chen Dongdong, Zheng Zhoushun, Wang Jianzhong et al. Rare Metals[J], 2016, 36: 1

5 Zhang Wen, Schneibel J H. Computational Materials Science[J], 1994, 3(3): 347

6 Yang Xiaohong, Sun Te, Xiao Peng et al. Rare Metal Materials and Engineering[J], 2013, 42(7): 1492 (in Chinese)

7 Shi Wei, Yang Bangchao, Zhang Xuanhong et al. Rare Metal Materials and Engineering [J], 2015, 44(2): 319

8 Tang Huiping, Wang Jianzhong, Ao Qingbo et al. Rare Metal Materials and Engineering[J], 2015, 44(8): 1821

9 Ao Qingbo, Tang Huiping, Wang Jianzhong et al. Rare Metal Materails and Engineering[J], 2014, 43(10): 2344

10 Zhang Wen. Applied Numerical Mathematics[J], 1996, 20(2): 235

11 Bruchon J, Drapier S, Valdivieso. International Journal for Numerical Methods in Engineering [J], 2011, 86(7): 845

12 Munoz P D, Bruchon J, Drapier S et al. International Journal for Numerical Methods in Engineering[J], 2013, 93(9): 919

13 Nichols F A, Mullins W W. Journal of Applied Physics[J], 1965, 36(6): 1826

14 Wakai F, Brakke K A. Acta Materialia[J], 2011, 59(14): 5379

15 Tritscher P, Broadbridge P. The Royal Society[J], 1995, 450(1940): 569

16 Fumihiro Wakai, Fritz Aldinger. Acta Materialia[J], 2003, 51(3): 641

17 Pan J, Cocks A C F. Acta metall Mater[J], 1995, 43(4): 1395

18 Zhang Wen, Schneibel J H. Acta Metall Mater[J], 1995, 43(12): 4377

19 Du Jixiang, Liang Shuhua, Wang Xianhui et al. Acta Metall $\operatorname{Sin}[\mathrm{J}], 2009,22(4): 263$

20 Zhang Wen, Gladwell Ian. Computational Materials Science[J], 1998, 12(2): 84

\title{
表面扩散机制下非等径金属粉末烧结过程的数值模拟研究
}

\author{
宋 敏 ${ }^{1}$, 郑洲顺 ${ }^{1}$, 谌东东 ${ }^{1}$, 汤慧萍 ${ }^{2}$, 王建忠 ${ }^{2}$ \\ (1. 中南大学, 湖南 长沙 410083)
}

(2. 西北有色金属研究院 金属多孔材料国家重点实验室，陕西 西安 710016)

\begin{abstract}
摘 要: 主要进行了表面扩散机制下非等径金属粉末烧结过程的数值模拟研究。为了准确地描述非等径金属粉末的烧结过程, 对表面扩 散模型建立了更加合适的初始边值条件, 并用有限差分法对该模型进行数值求解, 实现了半径比为 $1: 1 、 1: 1.5 、 1: 2$ 的非等径金属粉末烧 结过程的二维及三维数值模拟。非等径金属粉末烧结过程的数值模拟结果与实验结果吻合较好。最后, 分析了非等径金属粉末的半径比 对烧结颈半径生长速度的影响。数值模拟结果表明：随着金属粉末半径比的减小，烧结颈半径生长速度逐渐变快。
\end{abstract} 关键词: 烧结; 金属粉末; 表面扩散; 有限差分; 半径比 